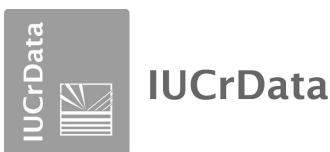

ISSN 2414-3146

Received 29 November 2018

Accepted 15 December 2018

Edited by M. Zeller, Purdue University, USA

Keywords: crystal structure; phenolic acid; 2,4dihydroxy-6-methylbenzoic acid ethyl ester.

CCDC reference: 1885274

Structural data: full structural data are available from iucrdata.iucr.org

\section{Crystal structure of the ethyl 2,4-dihydroxy-6- methylbenzoate from Illicium difengpi K.I.B et K.I.M.}

\author{
Huiping Xiong, ${ }^{\text {a }}$ Shilong Mao, ${ }^{\mathrm{b}}$ Qianzhou $\mathrm{Lv}^{\mathrm{b}}$ and Ning Zhang ${ }^{\mathrm{b} *}$
}

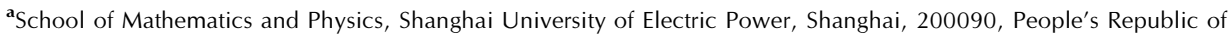
China, and 'bepartment of Pharmacy, Xuhui District Central Hospital, Shanghai, 200031, People's Republic of China. *Correspondence e-mail: zhangningpharm@163.com

The title compound, $\mathrm{C}_{10} \mathrm{H}_{12} \mathrm{O}_{4}$, was isolated from Illicium difengpi K.I.B et K.I.M. An intramolecular $\mathrm{O}-\mathrm{H}$... O hydrogen bond stabilizes the molecular conformation. In the crystal, the compound forms offset slanted stacks of alternating inversion-related molecules along the $a$ axis direction. Intermolecular $\mathrm{O}-\mathrm{H} \cdots \mathrm{O}$ hydrogen bonds link the molecules into double strands parallel to the [101] direction.
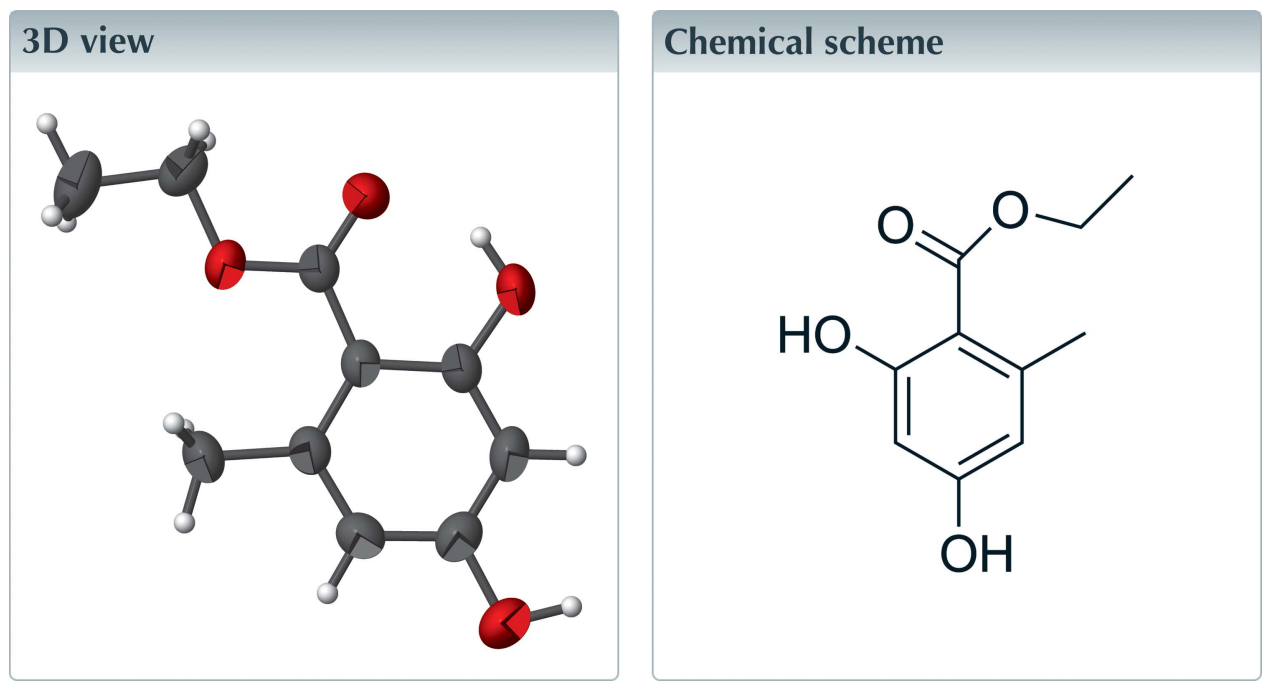

\section{Structure description}

Illicium difengpi is a small shrub growing in Guangxi province of China, belonging to the family Illiciaceae (He et al., 2014). The stem bark of I. difengpi is listed in the Chinese Pharmacopoeia (Pharmacopeia Committee of P. R. of China, 2010). It is an important traditional Chinese medicine and is mainly used as a treatment for rheumatic arthritics (Huang et al., 1996). The alcoholature of the stem bark of I. difengpi showed outstanding clinical efficacy and pharmacodynamics potency. Previous studies led to the isolation of phenylpropanoids, lignans, neolignans and triterpenoids from an extract of I. difengpi (Kouno et al., 1992,1993; Wang et al., 1994; Huang et al., 1997; Fang et al., 2010, 2011; Chu et al., 2011; Li et al., 2013, 2015a,b; Pan et al., 2016). An ongoing search for bioactive natural products from folk medicine resulted in the isolation of the title compound, which was previously obtained from Umbilicaria esculenta (Miyoshi) Minks (Qiu \& Ding, 2001). The isolation of the title compound from the stem bark of I. difengpi and its crystal structure are reported here.

The molecular structure of the title compound is shown in Fig. 1. The molecular structure contains a methyl group, two hydroxyl groups, and a carboethoxy group, which 
Table 1

Hydrogen-bond geometry $\left(\AA,^{\circ}\right)$.

\begin{tabular}{lllll}
\hline$D-\mathrm{H} \cdots A$ & $D-\mathrm{H}$ & $\mathrm{H} \cdots A$ & $D \cdots A$ & $D-\mathrm{H} \cdots A$ \\
\hline $\mathrm{O} 3-\mathrm{H} 3 \cdots \mathrm{O} 1$ & $0.89(2)$ & $1.72(3)$ & $2.4868(18)$ & $143(3)$ \\
$\mathrm{O}^{\mathrm{i}}-\mathrm{H} 4 \cdots 3^{\mathrm{i}}$ & $0.96(3)$ & $1.91(3)$ & $2.853(2)$ & $169(3)$ \\
$\mathrm{C}^{\mathrm{i}}-\mathrm{H} 3 A \cdots \mathrm{O}^{\mathrm{ii}}$ & 0.93 & 2.54 & $3.290(2)$ & 138 \\
\hline
\end{tabular}

Symmetry codes: (i) $x+\frac{1}{2},-y+\frac{1}{2}, z+\frac{1}{2}$; (ii) $x-\frac{1}{2},-y+\frac{1}{2}, z-\frac{1}{2}$.

are attached to $\mathrm{C} 2, \mathrm{C} 4, \mathrm{C} 6$ and $\mathrm{C} 7$ of the central benzene ring, respectively. An intramolecular $\mathrm{O} 3-\mathrm{H} 3 \cdots \mathrm{O} 1$ hydrogen bond (Table 1) stabilizes the molecular conformation.

In the crystal, $\mathrm{O} 4-\mathrm{H} 4 \cdots \mathrm{O} 3^{\mathrm{i}}$ and $\mathrm{C} 3-\mathrm{H} 3 A \cdots \mathrm{O} 4^{\mathrm{ii}}$ hydrogen bonds link the molecules into double strands parallel to the [101] direction. (Fig. 2, Table 2). In the solid state, the compound also forms offset slanted stacks of alternating inversion-related molecules along the $a$-axis direction. (Fig. 3)

A search of the Cambridge Crystallographic Database (version 5.39 with updates up to May 2018; Groom et al., 2016) indicated that no 2,4-dihydroxy-6-methylbenzoic acid ethyl ester has been structurally characterized. Four structurally similar 2,4-dihydroxy-6-methylbenzoic acid derivatives have been reported, namely (2-ethoxycarbonyl-3,5-dihydroxyphenyl)acetic acid monohydrate (Luck \& Mendenhall, 2002), phomozin monohydrate (Mazars et al.,1990), 2,3-dimethyl-3( $O$-orsellinoyl)lactic acid monohydrate (Declercq et al.,1991) and 2,3,4,5-tetrahydroxypentyl 4,6-dihydroxy-2,3-dimethylbenzoate (Talontsi et al., 2012).

\section{Synthesis and crystallization}

The stem bark of Illicium difengpi $(5.0 \mathrm{~kg})$, which was purchased from Caitongde Pharmacy, Shanghai, China, was powdered and extracted three times with aqueous ethanol (ethanol/water 8:2) under reflux. The solvent was then evaporated under reduced pressure to obtain a dry residue $(150 \mathrm{~g})$. The residue was suspended in water $(2 \mathrm{~L})$ and extracted successively with petroleum ether $(3 \times 2 \mathrm{~L})$, EtOAc $(3 \times 2 \mathrm{~L})$ and $\mathrm{BuOH}(3 \times 2 \mathrm{~L})$, affording $5 \mathrm{~g}, 70 \mathrm{~g}$, and $40 \mathrm{~g}$,

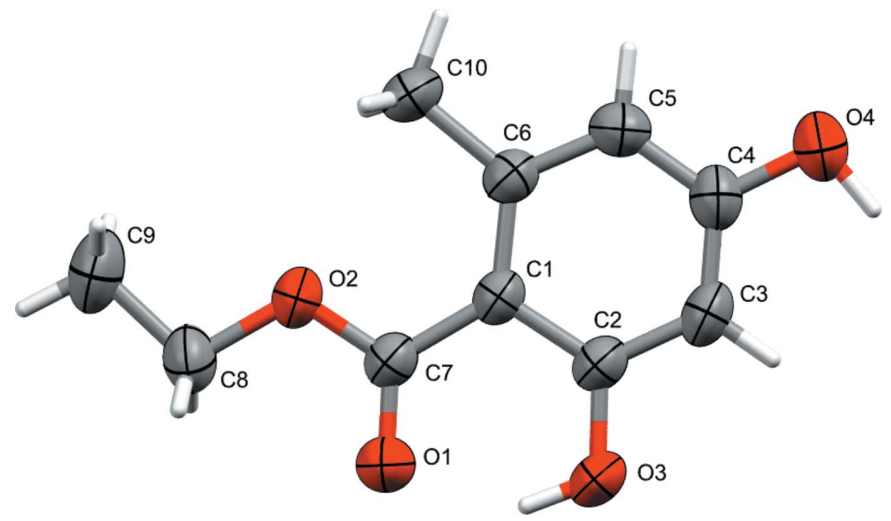

Figure 1

The molecular structure. Displacement ellipsoids are shown at the 50\% probability level.

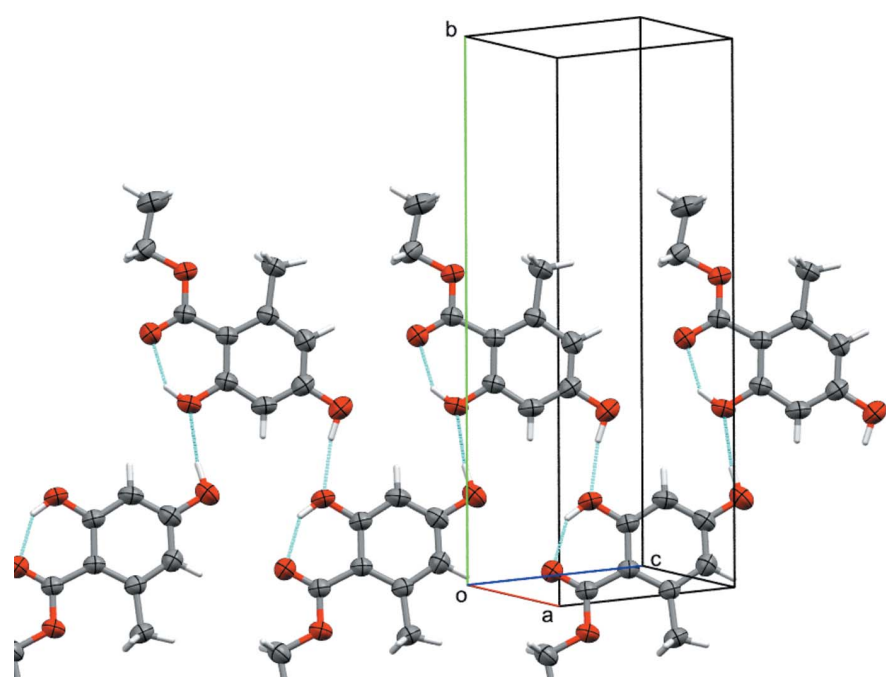

Figure 2

The packing of the title compound viewed along the $a$-axis direction. Dashed lines indicate hydrogen bonds.

respectively, of each dried fraction. The EtOAc fraction was subjected to silica gel column chromatography using gradient elution $\left(\mathrm{CH}_{2} \mathrm{Cl}_{2} / \mathrm{CH}_{3} \mathrm{OH}, 200: 1\right.$ to $\left.2: 1, v / v\right)$ to give four main fractions (Fr.1-1-Fr.1-4), of which Fr.1-2 was purified by successive silica gel column chromatography $\left(\mathrm{CH}_{2} \mathrm{Cl}_{2} /\right.$ $\left.\mathrm{CH}_{3} \mathrm{OH}, \quad 100: 1 \rightarrow 20: 1\right) ;$ 2,4-dihydroxy-6-methylbenzoic acid ethyl ester $(30 \mathrm{mg})$ was obtained from the fraction eluted by $\mathrm{CH}_{2} \mathrm{Cl}_{2} / \mathrm{CH}_{3} \mathrm{OH}$ (40:1). Single crystals suitable for X-ray diffraction analysis were obtained by slow evaporation from acetone solution after two weeks at room temperature.

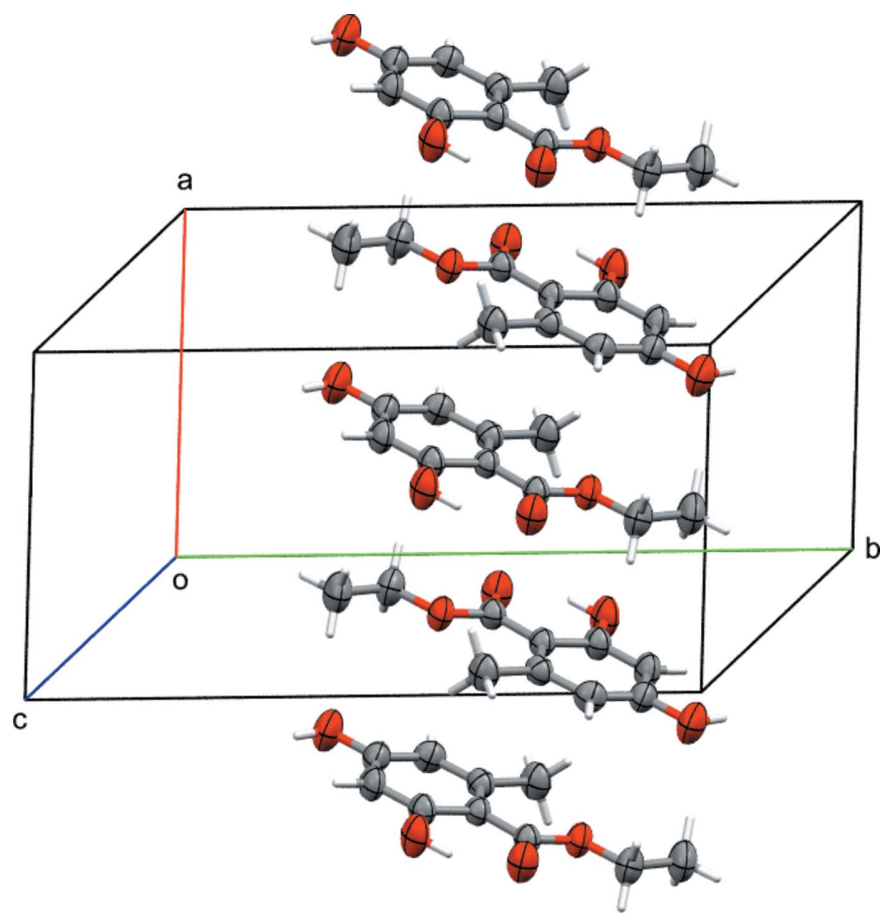

Figure 3

One of the slanted stacks of inversion-related molecules along the $a$-axis direction. 


\section{Refinement}

Crystal data, data collection and structure refinement details are summarized in Table 2.

\section{Acknowledgements}

The authors thank Professor Zhenxia Chen (Department of Chemistry, Fudan University, Shanghai) for the structure analysis.

\section{Funding information}

Funding for this research was provided by: Research Project of Xuhui District Central Hospital (award No. 201706).

\section{References}

Bruker (1997). SMART. Bruker AXS Inc., Madison, Wisconsin, USA.

Bruker (1999). SAINT-Plus. Bruker AXS Inc., Madison, Wisconsin, USA.

Chu, S.-S., Wang, C.-F., Du, S.-S., Liu, S.-L. \& Liu, Z.-L. (2011). J. Insect Sci. 11, 152.

Declercq, J.-P., Klaebe, A., Rossignol, M. \& Mazars, C. (1991). Acta Cryst. C47, 470-472.

Fang, L., Du, D., Ding, G.-Z., Si, Y.-K., Yu, S.-S., Liu, Y., Wang, W.-J., Ma, S.-G., Xu, S., Qu, J., Wang, J. M. \& Liu, Y. X. (2010). J. Nat. Prod. 73, 818-824.

Fang, L., Wang, X.-J., Ma, S.-G. \& Yu, S.-S. (2011). Acta Pharm. Sin. B, 1, 178-183.

Groom, C. R., Bruno, I. J., Lightfoot, M. P. \& Ward, S. C. (2016). Acta Cryst. B72, 171-179.

He, Y.-Z., Osoro, E. K., Palmer, S. I., Wang, L.-N. \& Aboud, N. S. (2014). Chinese Herbal Medicines, 6, 76-79.

Huang, P., Nishi, M., Zheng, X.-Z., Lai, M.-X. \& Naknishi, T. (1997). Acta Pharm. Sin, 32, 704-707.

Huang, P., Xi, Z.-M., Zheng, X.-Z., Lai, M.-X. \& Zhong, X.-Q. (1996). Acta Pharm. Sin, 31, 278-281.

Kouno, I., Yanagida, Y., Shimono, S., Shintomi, M., Ito, Y. \& ChunShu, Y. (1993). Phytochemistry, 32, 1573-1577.

Kouno, I., Yanagida, Y., Shimono, S., Shintomi, M. \& Yang, C.-S. (1992). Chem. Pharm. Bull. 40, 2461-2464.

Li, C.-T., Wu, Z.-J. \& Chen, W.-S. (2013). Evid-Based Compl. Alt, 2013, 942541.

Li, C.-T., Wu, Z.-J. \& Chen, W.-S. (2015a). Nat. Prod. Res. 29, $1793-$ 1797.

Li, C.-T., Wu, Z.-J. \& Chen, W.-S. (2015b). Rec. Nat. Prod, 9, 251261.

Luck, R. L. \& Mendenhall, G. D. (2002). Acta Cryst. E58, o1387o1388.
Table 2

Experimental details.

\begin{tabular}{|c|c|}
\hline \multicolumn{2}{|l|}{ Crystal data } \\
\hline Chemical formula & $\mathrm{C}_{10} \mathrm{H}_{12} \mathrm{O}_{4}$ \\
\hline$M_{\mathrm{r}}$ & 196.20 \\
\hline Crystal system, space group & Monoclinic, $P 2_{1} / n$ \\
\hline Temperature $(\mathrm{K})$ & 293 \\
\hline$a, b, c(\AA)$ & 7.818 (3), 17.017 (6), 8.189 (3) \\
\hline$\beta\left({ }^{\circ}\right)$ & $117.459(4)$ \\
\hline$V\left(\mathrm{~A}^{3}\right)$ & $966.7(6)$ \\
\hline$Z$ & 4 \\
\hline Radiation type & Mo $K \alpha$ \\
\hline$\mu\left(\mathrm{mm}^{-1}\right)$ & 0.10 \\
\hline Crystal size $(\mathrm{mm})$ & $0.15 \times 0.13 \times 0.08$ \\
\hline \multicolumn{2}{|l|}{ Data collection } \\
\hline Diffractometer & $\begin{array}{l}\text { Bruker SMART APEX CCD area- } \\
\text { detector }\end{array}$ \\
\hline Absorption correction & $\begin{array}{l}\text { Multi-scan (SAINT-Plus; Bruker, } \\
\text { 1999) }\end{array}$ \\
\hline$T_{\min }, T_{\max }$ & $0.405,0.968$ \\
\hline $\begin{array}{l}\text { No. of measured, independent and } \\
\text { observed }[I>2 \sigma(I)] \text { reflections }\end{array}$ & $4542,2097,1390$ \\
\hline$R_{\text {int }}$ & 0.037 \\
\hline$(\sin \theta / \lambda)_{\max }\left(\AA^{-1}\right)$ & 0.649 \\
\hline \multicolumn{2}{|l|}{ Refinement } \\
\hline$R\left[F^{2}>2 \sigma\left(F^{2}\right)\right], w R\left(F^{2}\right), S$ & $0.057,0.172,1.02$ \\
\hline No. of reflections & 2097 \\
\hline No. of parameters & 135 \\
\hline No. of restraints & 2 \\
\hline $\mathrm{H}$-atom treatment & $\begin{array}{l}\mathrm{H} \text { atoms treated by a mixture of } \\
\text { independent and constrained } \\
\text { refinement }\end{array}$ \\
\hline$\Delta \rho_{\max }, \Delta \rho_{\min }\left(\mathrm{e} \AA^{-3}\right)$ & $0.45,-0.23$ \\
\hline
\end{tabular}

Computer programs: SMART (Bruker, 1997), SAINT-Plus (Bruker, 1999), SHELXS97 and SHELXTL (Sheldrick, 2008), SHELXL2016 (Sheldrick, 2015) and publCIF (Westrip, 2010).

Mazars, C., Rossignol, M. P., Auriol, P. \& Klaebe, A. (1990). Phytochemistry, 29, 3441-3444.

Pan, Z.-H., Ning, D.-S., Huang, S.-S., Cheng, L., Xia, M.-W., Peng, L.Y. \& Li, D.-P. (2016). Molecules, 21, 607.

Pharmacopeia Committee of P. R. of China (2010). Pharmacopoeia of People's Republic of China. Beijing: Medical Science and Technology Press.

Qiu, C. \& Ding, Y. (2001). China J. Chin. Mater. Med, 26, 608-610.

Sheldrick, G. M. (2008). Acta Cryst. A64, 112-122.

Sheldrick, G. M. (2015). Acta Cryst. C71, 3-8.

Talontsi, F. M., Nwemeguela Kenla, T. J., Dittrich, B., Douanla-Meli, C. \& Laatsch, H. (2012). Planta Med. 78, 1020-1023.

Wang, J.-L., Yang, C.-S. \& Da, W.-R. (1994). China J. Chin. Mater. Med, 19, 422-423.

Westrip, S. P. (2010). J. Appl. Cryst. 43, 920-925. 


\section{full crystallographic data}

IUCrData (2018). 3, x181776 [https://doi.org/10.1107/S2414314618017765]

\section{Crystal structure of the ethyl 2,4-dihydroxy-6-methylbenzoate from Illicium difengpi K.I.B et K.I.M.}

Huiping Xiong, Shilong Mao, Qianzhou Lv and Ning Zhang

Ethyl 2,4-dihydroxy-6-methylbenzoate

\section{Crystal data}

$\mathrm{C}_{10} \mathrm{H}_{12} \mathrm{O}_{4}$

$M_{r}=196.20$

Monoclinic, $P 2_{1} / n$

$a=7.818(3) \AA$

$b=17.017(6) \AA$

$c=8.189$ (3) $\AA$

$\beta=117.459(4)^{\circ}$

$V=966.7(6) \AA^{3}$

$Z=4$

\section{Data collection}

Bruker SMART APEX CCD area-detector diffractometer

phi and $\omega$ scans

Absorption correction: multi-scan

(SAINT-Plus; Bruker, 1999)

$T_{\min }=0.405, T_{\max }=0.968$

4542 measured reflections

\section{Refinement}

Refinement on $F^{2}$

Least-squares matrix: full

$R\left[F^{2}>2 \sigma\left(F^{2}\right)\right]=0.057$

$w R\left(F^{2}\right)=0.172$

$S=1.02$

2097 reflections

135 parameters

2 restraints

Primary atom site location: structure-invariant direct methods
$F(000)=416$

$D_{\mathrm{x}}=1.348 \mathrm{Mg} \mathrm{m}^{-3}$

Mo $K \alpha$ radiation, $\lambda=0.71073 \AA$

Cell parameters from 878 reflections

$\theta=2.4-26.4^{\circ}$

$\mu=0.10 \mathrm{~mm}^{-1}$

$T=293 \mathrm{~K}$

Sheet, colorless

$0.15 \times 0.13 \times 0.08 \mathrm{~mm}$

2097 independent reflections

1390 reflections with $I>2 \sigma(I)$

$R_{\text {int }}=0.037$

$\theta_{\max }=27.5^{\circ}, \theta_{\min }=2.4^{\circ}$

$h=-5 \rightarrow 10$

$k=-21 \rightarrow 22$

$l=-10 \rightarrow 8$

Secondary atom site location: difference Fourier map

Hydrogen site location: mixed

$\mathrm{H}$ atoms treated by a mixture of independent and constrained refinement

$w=1 /\left[\sigma^{2}\left(F_{\mathrm{o}}{ }^{2}\right)+(0.1019 P)^{2}\right]$

where $P=\left(F_{\mathrm{o}}^{2}+2 F_{\mathrm{c}}{ }^{2}\right) / 3$

$(\Delta / \sigma)_{\max }<0.001$

$\Delta \rho_{\max }=0.45$ e $\AA^{-3}$

$\Delta \rho_{\min }=-0.23$ e $\AA^{-3}$

Special details

Geometry. All esds (except the esd in the dihedral angle between two 1.s. planes) are estimated using the full covariance matrix. The cell esds are taken into account individually in the estimation of esds in distances, angles and torsion angles; correlations between esds in cell parameters are only used when they are defined by crystal symmetry. An approximate (isotropic) treatment of cell esds is used for estimating esds involving l.s. planes. 
Refinement. The positions of hydroxyl $\mathrm{H}$ atoms attached to $\mathrm{O} 3$ and $\mathrm{O} 4$ were refined. All other $\mathrm{H}$ atoms were positioned geometrically and treated as riding atoms: $\mathrm{C}-\mathrm{H}=0.93-0.97 \AA . U_{\text {iso }}(\mathrm{H})$ were set to $1.5 U_{\mathrm{eq}}(\mathrm{C} / \mathrm{O})$ for $\mathrm{CH}_{3}$ and $\mathrm{OH}$, and to $1.2 U_{\mathrm{eq}}(\mathrm{C})$ for $\mathrm{CH}_{2}$ and aromatic $\mathrm{C}-\mathrm{H}$.

Fractional atomic coordinates and isotropic or equivalent isotropic displacement parameters $\left(\AA^{2}\right)$

\begin{tabular}{|c|c|c|c|c|}
\hline & $x$ & $y$ & $z$ & $U_{\text {iso }} * / U_{\text {eq }}$ \\
\hline $\mathrm{O} 1$ & $-0.0021(2)$ & $0.46363(7)$ & $-0.27773(18)$ & $0.0663(5)$ \\
\hline $\mathrm{O} 2$ & $0.11562(18)$ & $0.57656(6)$ & $-0.13811(16)$ & $0.0529(4)$ \\
\hline $\mathrm{O} 3$ & $0.1367(2)$ & $0.33529(7)$ & $-0.13142(19)$ & $0.0679(5)$ \\
\hline $\mathrm{H} 3$ & $0.050(4)$ & $0.3664(16)$ & $-0.216(3)$ & $0.102 *$ \\
\hline $\mathrm{O} 4$ & $0.6778(2)$ & $0.32885(8)$ & $0.44828(18)$ & $0.0718(5)$ \\
\hline $\mathrm{H} 4$ & $0.648(4)$ & $0.2753(16)$ & $0.410(4)$ & $0.108^{*}$ \\
\hline $\mathrm{C} 1$ & $0.2660(2)$ & $0.45967(8)$ & $0.0181(2)$ & $0.0420(4)$ \\
\hline $\mathrm{C} 2$ & $0.2682(2)$ & $0.37634(9)$ & $0.0131(2)$ & $0.0484(4)$ \\
\hline $\mathrm{C} 3$ & $0.4047(3)$ & $0.33288(9)$ & $0.1552(3)$ & $0.0551(5)$ \\
\hline $\mathrm{H} 3 \mathrm{~A}$ & 0.403637 & 0.278312 & 0.148712 & $0.066^{*}$ \\
\hline $\mathrm{C} 4$ & $0.5412(3)$ & $0.37051(10)$ & $0.3052(2)$ & $0.0524(5)$ \\
\hline $\mathrm{C} 5$ & $0.5453(3)$ & $0.45134(10)$ & $0.3168(2)$ & $0.0525(5)$ \\
\hline H5 & 0.640522 & 0.475644 & 0.420632 & $0.063 *$ \\
\hline C6 & $0.4098(2)$ & $0.49723(9)$ & $0.1763(2)$ & $0.0450(4)$ \\
\hline $\mathrm{C} 7$ & $0.1165(2)$ & $0.49885(9)$ & $-0.1435(2)$ & $0.0450(4)$ \\
\hline $\mathrm{C} 8$ & $-0.0274(3)$ & $0.61634(11)$ & $-0.3005(3)$ & $0.0612(5)$ \\
\hline $\mathrm{H} 8 \mathrm{~A}$ & -0.156271 & 0.600967 & -0.324130 & $0.073 *$ \\
\hline H8B & -0.010341 & 0.602890 & -0.407205 & $0.073 *$ \\
\hline $\mathrm{C} 9$ & $0.0009(4)$ & $0.70282(12)$ & $-0.2630(3)$ & $0.0802(7)$ \\
\hline H9A & -0.015784 & 0.715282 & -0.156911 & $0.120 *$ \\
\hline H9B & -0.091898 & 0.731300 & -0.367576 & $0.120^{*}$ \\
\hline H9C & 0.128638 & 0.717279 & -0.240524 & $0.120^{*}$ \\
\hline $\mathrm{C} 10$ & $0.4251(3)$ & $0.58478(9)$ & $0.2029(3)$ & $0.0592(5)$ \\
\hline $\mathrm{H} 10 \mathrm{~A}$ & 0.535726 & 0.597063 & 0.317280 & $0.089 *$ \\
\hline H10B & 0.310970 & 0.604287 & 0.205170 & $0.089^{*}$ \\
\hline $\mathrm{H} 10 \mathrm{C}$ & 0.438365 & 0.608915 & 0.103419 & $0.089 *$ \\
\hline
\end{tabular}

Atomic displacement parameters $\left(\AA^{2}\right)$

\begin{tabular}{lllllll}
\hline & $U^{11}$ & $U^{22}$ & $U^{33}$ & $U^{12}$ & $U^{13}$ & $U^{23}$ \\
\hline O1 & $0.0702(9)$ & $0.0471(7)$ & $0.0545(8)$ & $-0.0021(6)$ & $0.0056(7)$ & $-0.0024(5)$ \\
O2 & $0.0591(8)$ & $0.0349(6)$ & $0.0567(8)$ & $0.0057(5)$ & $0.0198(6)$ & $0.0033(5)$ \\
O3 & $0.0783(10)$ & $0.0327(6)$ & $0.0659(9)$ & $-0.0050(6)$ & $0.0105(7)$ & $-0.0064(5)$ \\
O4 & $0.0743(10)$ & $0.0545(8)$ & $0.0646(9)$ & $0.0156(7)$ & $0.0131(8)$ & $0.0103(6)$ \\
C1 & $0.0470(9)$ & $0.0329(8)$ & $0.0473(9)$ & $-0.0001(6)$ & $0.0228(8)$ & $-0.0009(6)$ \\
C2 & $0.0547(10)$ & $0.0351(8)$ & $0.0517(10)$ & $-0.0036(7)$ & $0.0214(8)$ & $-0.0036(7)$ \\
C3 & $0.0657(12)$ & $0.0326(8)$ & $0.0659(11)$ & $0.0043(8)$ & $0.0294(10)$ & $0.0027(8)$ \\
C4 & $0.0542(10)$ & $0.0455(9)$ & $0.0558(10)$ & $0.0095(8)$ & $0.0238(9)$ & $0.0080(8)$ \\
C5 & $0.0501(10)$ & $0.0517(10)$ & $0.0479(10)$ & $-0.0036(7)$ & $0.0159(8)$ & $-0.0053(7)$ \\
C6 & $0.0497(9)$ & $0.0377(8)$ & $0.0484(9)$ & $-0.0032(7)$ & $0.0234(8)$ & $-0.0029(7)$ \\
C7 & $0.0496(9)$ & $0.0357(8)$ & $0.0485(9)$ & $-0.0018(7)$ & $0.0217(8)$ & $-0.0015(7)$
\end{tabular}




\begin{tabular}{lllllll} 
C8 & $0.0674(12)$ & $0.0505(10)$ & $0.0580(11)$ & $0.0125(9)$ & $0.0223(10)$ & $0.0125(8)$ \\
C9 & $0.0965(17)$ & $0.0464(11)$ & $0.0998(17)$ & $0.0137(10)$ & $0.0471(14)$ & $0.0177(10)$ \\
C10 & $0.0657(12)$ & $0.0390(9)$ & $0.0612(11)$ & $-0.0087(8)$ & $0.0192(10)$ & $-0.0093(8)$ \\
\hline
\end{tabular}

Geometric parameters $\left(A,{ }^{\circ}\right)$

\begin{tabular}{|c|c|c|c|}
\hline $\mathrm{O} 1-\mathrm{C} 7$ & $1.220(2)$ & $\mathrm{C} 4-\mathrm{C} 5$ & $1.378(3)$ \\
\hline $\mathrm{O} 2-\mathrm{C} 7$ & $1.323(2)$ & $\mathrm{C} 5-\mathrm{C} 6$ & $1.390(2)$ \\
\hline $\mathrm{O} 2-\mathrm{C} 8$ & $1.451(2)$ & $\mathrm{C} 5-\mathrm{H} 5$ & 0.9300 \\
\hline $\mathrm{O} 3-\mathrm{C} 2$ & $1.3502(19)$ & $\mathrm{C} 6-\mathrm{C} 10$ & $1.502(2)$ \\
\hline $\mathrm{O} 3-\mathrm{H} 3$ & $0.89(2)$ & $\mathrm{C} 8-\mathrm{C} 9$ & $1.499(3)$ \\
\hline $\mathrm{O} 4-\mathrm{C} 4$ & $1.365(2)$ & $\mathrm{C} 8-\mathrm{H} 8 \mathrm{~A}$ & 0.9700 \\
\hline $\mathrm{O} 4-\mathrm{H} 4$ & $0.96(3)$ & $\mathrm{C} 8-\mathrm{H} 8 \mathrm{~B}$ & 0.9700 \\
\hline $\mathrm{C} 1-\mathrm{C} 6$ & $1.417(2)$ & C9-H9A & 0.9600 \\
\hline $\mathrm{C} 1-\mathrm{C} 2$ & $1.419(2)$ & C9-H9B & 0.9600 \\
\hline $\mathrm{C} 1-\mathrm{C} 7$ & $1.461(2)$ & $\mathrm{C} 9-\mathrm{H} 9 \mathrm{C}$ & 0.9600 \\
\hline $\mathrm{C} 2-\mathrm{C} 3$ & $1.377(2)$ & $\mathrm{C} 10-\mathrm{H} 10 \mathrm{~A}$ & 0.9600 \\
\hline $\mathrm{C} 3-\mathrm{C} 4$ & $1.359(2)$ & $\mathrm{C} 10-\mathrm{H} 10 \mathrm{~B}$ & 0.9600 \\
\hline $\mathrm{C} 3-\mathrm{H} 3 \mathrm{~A}$ & 0.9300 & $\mathrm{C} 10-\mathrm{H} 10 \mathrm{C}$ & 0.9600 \\
\hline $\mathrm{C} 7-\mathrm{O} 2-\mathrm{C} 8$ & $116.70(13)$ & $\mathrm{O} 1-\mathrm{C} 7-\mathrm{O} 2$ & $120.51(15)$ \\
\hline $\mathrm{C} 2-\mathrm{O} 3-\mathrm{H} 3$ & $112.1(18)$ & $\mathrm{O} 1-\mathrm{C} 7-\mathrm{C} 1$ & $123.39(15)$ \\
\hline $\mathrm{C} 4-\mathrm{O} 4-\mathrm{H} 4$ & $103.7(17)$ & $\mathrm{O} 2-\mathrm{C} 7-\mathrm{C} 1$ & $116.10(13)$ \\
\hline $\mathrm{C} 6-\mathrm{C} 1-\mathrm{C} 2$ & $117.54(14)$ & $\mathrm{O} 2-\mathrm{C} 8-\mathrm{C} 9$ & $106.95(15)$ \\
\hline $\mathrm{C} 6-\mathrm{C} 1-\mathrm{C} 7$ & $126.02(14)$ & $\mathrm{O} 2-\mathrm{C} 8-\mathrm{H} 8 \mathrm{~A}$ & 110.3 \\
\hline $\mathrm{C} 2-\mathrm{C} 1-\mathrm{C} 7$ & $116.42(14)$ & $\mathrm{C} 9-\mathrm{C} 8-\mathrm{H} 8 \mathrm{~A}$ & 110.3 \\
\hline $\mathrm{O} 3-\mathrm{C} 2-\mathrm{C} 3$ & $116.31(14)$ & $\mathrm{O} 2-\mathrm{C} 8-\mathrm{H} 8 \mathrm{~B}$ & 110.3 \\
\hline $\mathrm{O} 3-\mathrm{C} 2-\mathrm{C} 1$ & $121.87(14)$ & $\mathrm{C} 9-\mathrm{C} 8-\mathrm{H} 8 \mathrm{~B}$ & 110.3 \\
\hline $\mathrm{C} 3-\mathrm{C} 2-\mathrm{C} 1$ & $121.82(15)$ & $\mathrm{H} 8 \mathrm{~A}-\mathrm{C} 8-\mathrm{H} 8 \mathrm{~B}$ & 108.6 \\
\hline $\mathrm{C} 4-\mathrm{C} 3-\mathrm{C} 2$ & $119.36(15)$ & $\mathrm{C} 8-\mathrm{C} 9-\mathrm{H} 9 \mathrm{~A}$ & 109.5 \\
\hline $\mathrm{C} 4-\mathrm{C} 3-\mathrm{H} 3 \mathrm{~A}$ & 120.3 & $\mathrm{C} 8-\mathrm{C} 9-\mathrm{H} 9 \mathrm{~B}$ & 109.5 \\
\hline $\mathrm{C} 2-\mathrm{C} 3-\mathrm{H} 3 \mathrm{~A}$ & 120.3 & $\mathrm{H} 9 \mathrm{~A}-\mathrm{C} 9-\mathrm{H} 9 \mathrm{~B}$ & 109.5 \\
\hline $\mathrm{C} 3-\mathrm{C} 4-\mathrm{O} 4$ & $120.56(15)$ & $\mathrm{C} 8-\mathrm{C} 9-\mathrm{H} 9 \mathrm{C}$ & 109.5 \\
\hline $\mathrm{C} 3-\mathrm{C} 4-\mathrm{C} 5$ & $121.07(15)$ & $\mathrm{H} 9 \mathrm{~A}-\mathrm{C} 9-\mathrm{H} 9 \mathrm{C}$ & 109.5 \\
\hline $\mathrm{O} 4-\mathrm{C} 4-\mathrm{C} 5$ & $118.36(16)$ & $\mathrm{H} 9 \mathrm{~B}-\mathrm{C} 9-\mathrm{H} 9 \mathrm{C}$ & 109.5 \\
\hline $\mathrm{C} 4-\mathrm{C} 5-\mathrm{C} 6$ & $121.27(16)$ & $\mathrm{C} 6-\mathrm{C} 10-\mathrm{H} 10 \mathrm{~A}$ & 109.5 \\
\hline $\mathrm{C} 4-\mathrm{C} 5-\mathrm{H} 5$ & 119.4 & $\mathrm{C} 6-\mathrm{C} 10-\mathrm{H} 10 \mathrm{~B}$ & 109.5 \\
\hline $\mathrm{C} 6-\mathrm{C} 5-\mathrm{H} 5$ & 119.4 & $\mathrm{H} 10 \mathrm{~A}-\mathrm{C} 10-\mathrm{H} 10 \mathrm{~B}$ & 109.5 \\
\hline $\mathrm{C} 5-\mathrm{C} 6-\mathrm{C} 1$ & $118.93(15)$ & $\mathrm{C} 6-\mathrm{C} 10-\mathrm{H} 10 \mathrm{C}$ & 109.5 \\
\hline $\mathrm{C} 5-\mathrm{C} 6-\mathrm{C} 10$ & $117.23(15)$ & $\mathrm{H} 10 \mathrm{~A}-\mathrm{C} 10-\mathrm{H} 10 \mathrm{C}$ & 109.5 \\
\hline $\mathrm{C} 1-\mathrm{C} 6-\mathrm{C} 10$ & $123.84(14)$ & $\mathrm{H} 10 \mathrm{~B}-\mathrm{C} 10-\mathrm{H} 10 \mathrm{C}$ & 109.5 \\
\hline $\mathrm{C} 6-\mathrm{C} 1-\mathrm{C} 2-\mathrm{O} 3$ & $179.79(15)$ & $\mathrm{C} 2-\mathrm{C} 1-\mathrm{C} 6-\mathrm{C} 5$ & $0.4(2)$ \\
\hline $\mathrm{C} 7-\mathrm{C} 1-\mathrm{C} 2-\mathrm{O} 3$ & $-1.4(2)$ & $\mathrm{C} 7-\mathrm{C} 1-\mathrm{C} 6-\mathrm{C} 5$ & $-178.29(16)$ \\
\hline $\mathrm{C} 6-\mathrm{C} 1-\mathrm{C} 2-\mathrm{C} 3$ & $-0.3(2)$ & $\mathrm{C} 2-\mathrm{C} 1-\mathrm{C} 6-\mathrm{C} 10$ & $-179.25(15)$ \\
\hline $\mathrm{C} 7-\mathrm{C} 1-\mathrm{C} 2-\mathrm{C} 3$ & $178.47(16)$ & $\mathrm{C} 7-\mathrm{C} 1-\mathrm{C} 6-\mathrm{C} 10$ & $2.1(3)$ \\
\hline $\mathrm{O} 3-\mathrm{C} 2-\mathrm{C} 3-\mathrm{C} 4$ & $-179.87(17)$ & $\mathrm{C} 8-\mathrm{O} 2-\mathrm{C} 7-\mathrm{O} 1$ & $-2.1(2)$ \\
\hline $\mathrm{C} 1-\mathrm{C} 2-\mathrm{C} 3-\mathrm{C} 4$ & $0.3(3)$ & $\mathrm{C} 8-\mathrm{O} 2-\mathrm{C} 7-\mathrm{C} 1$ & $177.74(14)$ \\
\hline
\end{tabular}


data reports

$\begin{array}{llll}\mathrm{C} 2-\mathrm{C} 3-\mathrm{C} 4-\mathrm{O} 4 & 179.75(17) & \mathrm{C} 6-\mathrm{C} 1-\mathrm{C} 7-\mathrm{O} 1 & 178.68(16) \\ \mathrm{C} 2-\mathrm{C} 3-\mathrm{C} 4-\mathrm{C} 5 & -0.2(3) & \mathrm{C} 2-\mathrm{C} 1-\mathrm{C} 7-\mathrm{O} 1 & 0.0(2) \\ \mathrm{C} 3-\mathrm{C} 4-\mathrm{C} 5-\mathrm{C} 6 & 0.3(3) & \mathrm{C} 6-\mathrm{C} 1-\mathrm{C} 7-\mathrm{O} 2 & -1.1(2) \\ \mathrm{O} 4-\mathrm{C} 4-\mathrm{C} 5-\mathrm{C} 6 & -179.68(16) & \mathrm{C} 2-\mathrm{C} 1-\mathrm{C} 7-\mathrm{O} 2 & -179.83(14) \\ \mathrm{C} 4-\mathrm{C} 5-\mathrm{C} 6-\mathrm{C} 1 & -0.4(2) & \mathrm{C} 7-\mathrm{O} 2-\mathrm{C} 8-\mathrm{C} 9 & -179.38(14) \\ \mathrm{C} 4-\mathrm{C} 5-\mathrm{C} 6-\mathrm{C} 10 & 179.29(16) & & \end{array}$

Hydrogen-bond geometry $\left(A,{ }^{\circ}\right)$

\begin{tabular}{lllll}
\hline$D-\mathrm{H} \cdots A$ & $D-\mathrm{H}$ & $\mathrm{H} \cdots A$ & $D \cdots A$ & $D-\mathrm{H} \cdots A$ \\
\hline $\mathrm{O} 3-\mathrm{H} 3 \cdots \mathrm{O} 1$ & $0.89(2)$ & $1.72(3)$ & $2.4868(18)$ & $143(3)$ \\
$\mathrm{O} 4-\mathrm{H} 4 \cdots \mathrm{O} 33^{\mathrm{i}}$ & $0.96(3)$ & $1.91(3)$ & $2.853(2)$ & $169(3)$ \\
$\mathrm{C} 3-\mathrm{H} 3 A \cdots \mathrm{O} 44^{\mathrm{ii}}$ & 0.93 & 2.54 & $3.290(2)$ & 138 \\
\hline
\end{tabular}

Symmetry codes: (i) $x+1 / 2,-y+1 / 2, z+1 / 2$; (ii) $x-1 / 2,-y+1 / 2, z-1 / 2$. 